\title{
Mixed Boundary Value Problems for Nonlinear Elliptic Systems in $n$-Dimensional Lipschitzian Domains
}

\author{
C. Ebmeyer
}

Abstract. Let $u: \Omega \rightarrow \mathbb{R}^{N}$ be the solution of the nonlinear elliptic system

$$
-\sum_{i=1}^{n} \partial_{i} F_{i}(x, \nabla u)=f(x)+\sum_{i=1}^{n} \partial_{i} f_{i}(x),
$$

where $\Omega \subset \mathbb{R}^{n}$ is a bounded domain with a piecewise smooth boundary (e.g., $\Omega$ is a polyhedron). It is assumed that a mixed boundary value condition is given. Global regularity results in Sobolev and in Nikolskii spaces are proven, in particular $\left[W^{r, 2}(\Omega)\right]^{N}$-regularity $\left(s<\frac{3}{2}\right)$ of $u$.

Keywords: Mixed boundary value problems, piecewise smooth boundaries, Nikolskii spaces AMS subject classification: Primary $35 \mathrm{~J} 55,35 \mathrm{~J} 65$, secondary $35 \mathrm{~J} 25$

\section{Introduction}

We treat the nonlinear elliptic system

$$
\left.\begin{array}{rlrl}
-\sum_{i=1}^{n} \partial_{i} F_{i}(x, \nabla u) & =f(x)+\sum_{i=1}^{n} \partial_{i} f_{i}(x) & & \text { in } \Omega \\
u(x) & =0 & & \text { on } \Gamma_{\mathcal{D}} \\
-\sum_{i=1}^{n} F_{i}(x, \nabla u) \nu_{i} & =\sum_{i=1}^{n} f_{i} \nu_{i} & & \text { on } \Gamma_{\mathcal{N}}
\end{array}\right\}
$$

where $\Omega \subset \mathbb{R}^{n}(n \geq 3)$ is bounded, $u: \Omega \rightarrow \mathbb{R}^{N}$ is a vector-valued function, $\partial_{i}=\frac{\partial}{\partial x_{i}}$, $\partial \Omega=\Gamma_{\mathcal{D}} \cup \Gamma_{\mathcal{N}}$ where $\Gamma_{\mathcal{D}}$ is the Dirichlet boundary and $\Gamma_{\mathcal{N}}$ is the Neumann boundary, and $\nu$ is the outward normal of $\partial \Omega$. We suppose that $\partial \Omega$ is piecewise smooth (e.g., $\Omega$ is a polyhedron or has a Lipschitz boundary).

In this paper we investigate the regularity of the solution $u$ of (0.1). Refining the method of [8] we obtain regularity results in Nikolskii spaces and in Sobolev spaces $\left[W^{s, 2}(\Omega)\right]^{N}$, especially $\left[W^{s, 2}(\Omega)\right]^{N}$-regularity $\left(s<\frac{3}{2}\right)$ of $u$ up to the boundary.

C. Ebmeyer: Universität Bonn, Mathematisches Seminar, NuBallee 15, D - 53115 Bonn

ISSN 0232-2064 / \$2.50 C Heldermann Verlag Berlin 
Solutions of mixed boundary value problems in non-smooth domains may have singularities on the boundary at such points where the boundary condition is changing or where $\partial \Omega$ is not smooth.

In the case of a linear elliptic equation various authors have investigated the regularity of the solution. They have given a decomposition of the solution $u$ into a regular and a singular part.In particular, for $\Omega \subset \mathbb{R}^{2}$. this provides an explicit description of the behaviour of $u$ near the boundary (cf. $[4,7,9,11]$ ). In the case when $\Omega \subset \mathbb{R}^{n}(n \geq 3)$ there are difficulties by finding such a decomposition which describes all the singularities of $u$ (see $[2,3,10,14,17])$.

In the case of nonlinear equations there are only few results. Semilinear Dirichlet problems on corner domains are treated in $[12,15]$ and in $[5,6]$, where results in weighted Sobolev spaces are given. Further, nonlinear mixed boundary value problems are investigated in [8]. Regularity results in Sobolev spaces are proven.

In this paper we generalize some results given in [8]. Let the boundary of $\Omega$ consist of smooth $(n-1)$-dimensional manifolds with piecewise smooth boundaries such that each boundary manifold is either a Dirichlet or a Neumann boundary manifold. Let us fix some point $P \in \partial \Omega$. Then we suppose that there is a ball $B(P)$ and a smooth mapping which maps $\Omega$ onto a domain $\hat{\Omega}$ such that $B(P) \cap \hat{\Omega}$ is the intersection of $B(P)$ and a polyhedron. In contrast to [8] we consider the case that $B(P) \cap \partial \hat{\Omega}$ contains more than one Dirichlet boundary manifold. Further, we admit that $B(P) \cap \hat{\Omega}$ is probably not convex. But we assume that each inner angle between a Dirichlet and a Neumann boundary manifold is not greater than $\pi$.

We suppose that there is a function $F(x, p)$ such that $F_{i}^{r}(x, p)$ is the partial derivative of $F(\dot{x}, p)$ with respect to the component corresponding to $p_{i}^{r}$ (here $F_{i}^{r}(x, p)$ denotes the $r$-th component of the vector $\left.F_{i}(x, p)\right)$. Hence, we deal with the variational case.

The aim of this paper is to show that $u \in\left[W^{9,2}(\Omega)\right]^{N}$ for $s<\frac{3}{2}$. This result is the best possible, for we admit that $\hat{\Omega}$ can be a polyhedron where the inner angle between a Dirichlet and a Neumann boundary manifold is equal to $\pi$. Otherwise, if all such angles are less than $\pi$, we prove that $u \in \mathcal{H}^{\frac{3}{2}, 2}(\Omega)$, where $\mathcal{H}^{s, p}(\Omega)$ denotes a Nikolskii space. Moreover, in the case when $N=1$ the solution $u$ of equation (0.1) is Hölder continuous. Then we show that $u \in L^{p}(\Omega)$ for some $p>3$.

This paper is organized as follows. In Section 1 we state the assumptions on the data and the main results. Section 2 contains notations. In Section 3 the proofs of the main results are given. Finally, in Section 4 we explain the proofs with examples of tree-dimensional domains.

\section{Assumptions on the data and main results}

We need the following assumptions on the data.

(A1) $\Omega \subset \mathbb{R}^{n} \quad(n \geq 3)$ is a connected open domain with Lipschitz boundary.

(A2) $\partial \Omega=\bigcup_{1 \leq i \leq M} \bar{\Gamma}_{i}$, where $\Gamma_{i}$ are open $(n-1)$-dimensional_manifolds, and $\Gamma_{i} \cap \Gamma_{j}=\emptyset$ holds for $i \neq j$. 
(A3) $\partial \Gamma_{i}(1 \leq i \leq M)$ are $(n-2)$-dimensional Lipschitz continuous manifolds.

(A4) $\Gamma_{1}, \ldots, \Gamma_{\sigma} \subset \Gamma_{\mathcal{D}}$ and $\Gamma_{\sigma+1}, \ldots, \Gamma_{M} \subset \Gamma_{\mathcal{N}}$.

(A5) $P \in \bigcap_{i \in \Lambda} \partial \Gamma_{i}$ implies that $|\Lambda| \leq n$.

(A6) To each point $P \in \partial \Omega$ there exists a mapping $\phi$ and a ball $B_{R}(\phi(P))$ such that:

(i) $B_{R}(\phi(P)) \cap \phi(\partial \Omega)$ is the intersection of $B_{R}(\phi(P))$ and a polyhedron.

(ii) $B_{R}(\phi(P)) \cap \phi(\partial \Omega)$ is simply connected.

(iii) $\phi, \phi^{-1} \in W_{\text {loc }}^{2, \infty}\left(\mathbb{R}^{n}\right)$ and the Jacobian of $\phi$ is positive definite.

(iv) If $\Gamma_{i} \in \Gamma_{\mathcal{D}}, \Gamma_{j} \in \Gamma_{\mathcal{N}}$, and $\partial \Gamma_{i} \cap \partial \Gamma_{j} \neq \emptyset$, then angle $\left(\phi\left(\Gamma_{i}\right), \phi\left(\Gamma_{j}\right)\right) \leq \pi$.

(v) At most one pair of boundary manifolds $\Gamma_{i}, \Gamma_{j}\left(i \neq j, \partial \Gamma_{i} \cap \partial \Gamma_{j} \neq \emptyset\right)$ satisfies angle $\left(\phi\left(\Gamma_{i}\right), \phi\left(\Gamma_{j}\right)\right)=\pi$.

\section{Remark.}

(i) By angle $\left(\phi\left(\Gamma_{i}\right), \phi\left(\Gamma_{j}\right)\right)$ we denote the inner angle between $\phi\left(\Gamma_{i}\right) \cap B_{R}(\phi(P))$ and $\phi\left(\Gamma_{j}\right) \cap B_{R}(\phi(P))$ where it is assumed that $\phi\left(\Gamma_{i}\right) \cap B_{R}(\phi(P)) \neq \emptyset$ and $\phi\left(\Gamma_{j}\right) \cap B_{R}(\phi(P)) \neq$ $\emptyset$.

(ii) We assume that the inner angle between a boundary manifold of $\phi\left(\Gamma_{\mathcal{D}}\right)$ and another one of $\phi\left(\Gamma_{\mathcal{N}}\right)$ is not greater than $\pi$ (cf. assumption (A6)/(ii)). But it is admitted that the inner angle between two boundary manifolds is greater than $\pi$ if there is no change of the boundary value condition.

(iii) It is also possible to treat domains with a slit. Then instead of assumption $(\mathrm{A} 6) /(\mathrm{v})$ we need the assumption that at most one pair of boundary manifolds $\Gamma_{i}, \Gamma_{j} \quad\left(i \neq j, \partial \Gamma_{i} \cap \partial \Gamma_{j} \neq \emptyset\right)$ satisfies angle $\left(\phi\left(\Gamma_{i}\right), \phi\left(\Gamma_{j}\right)\right)=\mu \pi, \mu \in\{1,2\}$.

Let $x \in \bar{\Omega}$ and $p \in \mathbb{R}^{n N}$ with components $x_{i} \quad(1 \leq i \leq n)$ and $p_{i}^{r}(1 \leq r \leq N)$, respectively. We suppose that there is a $C^{2}$-function $F(x, p): \Omega \times \mathbb{R}^{n N} \rightarrow \mathbb{R}$ such that $\frac{\partial}{\partial p_{i}^{r}} F(x, p)=F_{i}^{r}(x, p)$ for all $1 \leq i \leq n$ and $1 \leq r \leq N$, where $F_{i}^{r}(x, p)$ denotes the $r$-th component of $F_{i}(x, p) \in \mathbb{R}^{N}$. We set

$$
F_{x_{i}}(x, p)=\frac{\partial}{\partial x_{i}} F(x, p), \quad F_{i, x_{k}}(x, p)=\frac{\partial}{\partial x_{k}} F_{i}(x, p), \quad F_{i, k}^{r s}(x, p)=\frac{\partial}{\partial p_{k}^{s}} F_{i}^{r}(x, p)
$$

for $1 \leq i, k \leq n$ and $1 \leq r, s \leq N$. Furthermore, we suppose that there are functions $g_{0}, g_{x_{i}}, g_{i}$ and $g_{i, x_{k}}(1 \leq i, k \leq n)$ such that:

(H1) $c_{0}+c_{0}^{\prime}|p|^{2} \leq F(x, p) \leq g_{0}(x)+c|p|^{2}$ for $g_{0} \in L^{\infty}(\Omega)$ and $c_{0}^{\prime}>0$.

(H2) $\left|F_{x_{i}}(x, p)\right| \leq g_{x_{i}}(x)+c|p|^{2}$ for $g_{x_{i}} \in L^{1}(\Omega)$.

(H3) $\left|F_{i}(x, p)\right| \leq g_{i}(x)+c|p|$ for $g_{i} \in L^{2}(\Omega)$.

(H4) $\left|F_{i, x_{k}}(x, p)\right| \leq g_{i, x_{k}}(x)+c|p|$ for $g_{i, x_{k}} \in L^{2}(\Omega)$.

(H5) $\left|F_{i, k}^{r s}(x, p)\right| \leq c$.

(H6) There is a constant $k_{0}>0$ independent of $x$ and $p$ such that for all $\xi \in \mathbb{R}^{n N}$

$$
k_{0}|\xi|^{2} \leq \sum_{r, s=1}^{N} \sum_{i, k=1}^{n} F_{i, k}^{r s}(x, p) \xi_{i}^{r} \xi_{k}^{s}
$$

(H7) $f^{r}(x) \in L^{2}(\Omega)$ and $f_{i}^{r}(x) \in W^{1,2}(\Omega) \cap L^{\infty}(\Omega)$ for $1 \leq i \leq n$ and $1 \leq r \leq N$. 
Remark. Hypothesis (H6) can be replaced by the weaker condition

(H6') There are constants $k_{0}>0$ and $k_{1}$ independent of $x$ and $p$ such that for all $\xi \in\left[H^{1}(\Omega)\right]^{N}$

$$
k_{0} \int_{\Omega}|\nabla \xi|^{2} d x-k_{1} \int_{\Omega}|\xi|^{2} d x \leq \int_{\Omega} \sum_{r, s=1}^{N} \sum_{i, k=1}^{n} F_{i, k}^{r s}(x, \nabla u) \partial_{i} \xi^{r} \partial_{k} \xi^{s} d x
$$

Let us note that the changes to be made in the proofs are obvious.

Under the above hypotheses there exists a unique weak solution $u \in\left[W^{1,2}(\Omega)\right]^{N}$ of problem (0.1) (see [16]).

We use the usual Sobolev spaces $W^{s, p}(\Omega)$ and the Nikolskii spaces $\mathcal{H}^{s, p}(\Omega)$ (cf. [1]). In detail, let $s$ be no integer, let $z \in \mathbb{R}^{n}, s=m+\sigma$ where $0<\sigma<1$ and $m$ is an integer, $\Omega_{\eta}=\{x \in \Omega: \operatorname{dist}(x, \partial \Omega) \geq \eta\}$, and $1 \leq p<\infty$. The spaces $W^{s, p}(\Omega)$ and $\mathcal{H}^{s, p}(\Omega)$ consist of all functions $u$ for which the norms

$$
\|u\|_{W^{\prime, p}(\Omega)}=\left(\|u\|_{W^{m, p}(\Omega)}^{p}+\sum_{|\alpha|=m} \int_{\Omega} \int_{\Omega} \frac{\left|\partial^{\alpha} u(x)-\partial^{\alpha} u(y)\right|^{p}}{|x-y|^{n+p \sigma}} d x d y\right)^{\frac{1}{p}}
$$

and

$$
\|u\|_{\mathcal{H} \cdot \boldsymbol{p}(\Omega)}=\left(\|u\|_{L^{p}(\Omega)}^{p}+\sum_{|\alpha|=m} \sup _{\substack{\eta>0 \\ 0<|x|<\eta}} \int_{\Omega_{\eta}} \frac{\left|\partial^{\alpha} u(x+z)-\partial^{\alpha} u(x)\right|^{p}}{|z|^{\sigma p}} d x\right)^{\frac{1}{p}}
$$

are finite.

We will prove the following results:

Theorem 1.1.

a) The solution $u$ of equation (0.1) satisfies

$$
u \in\left[W^{s, 2}(\Omega)\right]^{N .} \quad \text { for all } s<\frac{3}{2} .
$$

b) If angle $\left(\Gamma_{i}, \Gamma_{j}\right) \neq \pi$ for each pair of boundary manifolds $\Gamma_{i}, \Gamma_{j}\left(i \neq j, \partial \Gamma_{i} \cap \partial \Gamma_{j} \neq\right.$ 0), then

$$
u \in\left[\mathcal{H}^{\frac{3}{2}, 2}(\Omega)\right]^{N}
$$

holds.

\section{Remark.}

(i) By assumption we consider the case when $n \geq 3$. But our proofs of (1.1) and (1.2) also hold when $n=2$.

(ii) angle $\left(\Gamma_{i}, \Gamma_{j}\right) \neq \pi$ implies that angle $\left(\phi\left(\Gamma_{i}\right), \phi\left(\Gamma_{j}\right)\right) \neq \pi$, for $\phi$ is smooth.

Using the Sobolev imbedding theorem and (1.1) we get $u \in\left[W^{1, s}(\Omega)\right]^{N}$ for $s<\frac{2 n}{n-1}$. Let us note that $\underline{s} \leq \underline{3 \text { for }} n \geq \geq 3$. The_next_theorem improves_this_result_in_the-case when $N=1$. 
Theorem 1.2. Let $N=1$ and let the functions $g_{x_{i}}, g_{i}, g_{i, x_{k}}, f$ and $f_{k}$ given in hypotheses (H1) - (H7) satisfy

$$
g_{i} \in L^{\frac{n}{1-6}}(\Omega), \quad g_{x_{i}}, g_{i, x_{k}}, f, \partial_{i} f_{k} \in L^{\frac{2 n}{3-3}}(\Omega)
$$

for $1 \leq i, k \leq n$ and some $\delta>0$. Then there exists a constant $\varepsilon_{0}>0$ independent of $n$ such that the solution $u$ of equation (0.1) satisfies

$$
\nabla u \in L^{s}(\Omega) \quad \text { for } s=3+\varepsilon_{0} .
$$

Remark. The results of Theorem 1.1 and Theorem 1.2 also hold for solutions $u(x, t)$ of parabolic systems. Let $u(x, 0) \in\left[W^{1,2}(\Omega)\right]^{N}$. Then we get the results (1.1), $(1.2)$, and $(1.4)$ in the spaces $\left[L^{2}\left(0, T ; W^{s, p}(\Omega)\right)\right]^{N}$ and $\left[L^{2}\left(0, T ; \mathcal{H}^{s, p}(\Omega)\right)\right]^{N}$.

\section{Notations}

Let $B_{R}(x)=\left\{y \in \mathbb{R}^{n}:|x-y|<R\right\}$. The boundary of $\Omega$ is piecewise smooth. By assumption to each point $P \in \partial \Omega$ there is a constant $R_{0}>0$ and a $W^{2, \infty}$-mapping

$$
\phi^{*}: x \rightarrow \hat{x}
$$

such that $B_{R_{0}}(\hat{P}) \cap \hat{\Omega}$ is the intersection of $B_{R_{0}}(\hat{P})$ and a polyhedron. (We use the denotations $\hat{P}=\phi^{*}(P), \hat{\Omega}=\phi^{*}(\Omega)$ etc. and we will write $B_{R}$ instead of $B_{R}(\hat{P})$.)

In the sequel we suppose that $\hat{P}$ and $R_{0} \in(0,1]$ are fixed such that $\hat{P}$ is the only vertex of $B_{R_{0}}(\hat{P}) \cap \partial \hat{\Omega}$ or that there is no vertex of $\partial \hat{\Omega}$ in $B_{R_{0}}(\hat{P})$. Further, let $\hat{P} \in \partial \hat{\Gamma}_{k}$ for some $k \in\{1, \ldots, M\}$.

We need appropriate basis vectors $\left\{\zeta^{1}, \ldots, \zeta^{n}\right\}$ in $B_{R_{0}}(\hat{P})$. Let $\Lambda_{1}, \Lambda_{2}$, and $\Lambda_{3}$ be disjoint index sets (some of them possibly empty) such that $\cup_{i=1}^{3} \Lambda_{i}=\{1, \ldots, n\}$. Let $\alpha^{*}>0,\left|\zeta^{i}\right|=1$ for $1 \leq i \leq n$, and angle $\left(\zeta^{i}, \zeta^{j}\right) \geq \alpha^{*}$ for $1 \leq i<j \leq n$. We assume the following:

1) $y+s \zeta^{i} \in(\hat{\Omega} \cup \partial \hat{\Omega})$ for $y \in\left(\partial \hat{\Omega} \cap B_{R_{0}}\right), 0<s<R_{0}$, and $1 \leq i \leq n$.

2) If $\hat{\Gamma}_{\mathcal{D}} \cap B_{R_{0}} \neq \emptyset$, then $\zeta^{i}\left(i \in \Lambda_{1}\right)$ is parallel to $\hat{\Gamma}_{\mathcal{D}} \cap B_{R_{0}}$.

3) If $\hat{\Gamma}_{\mathcal{D}} \cap B_{R_{0}}=\emptyset$, then $\Lambda_{1}=\{1, \ldots, n\}$.

4) If $i \in \Lambda_{1}, y \in\left(\hat{\Gamma}_{\mathcal{D}} \cap B_{R_{0}}\right), s>0$, and $y+s \zeta^{i} \in B_{R_{0}}$, then $y+s \zeta^{i} \in \hat{\Gamma}_{\mathcal{D}}$.

5) If $\hat{\Gamma}_{\mathcal{N}} \cap B_{R_{0}} \neq \emptyset$, then $\zeta^{i}\left(i \in \Lambda_{2}\right)$ is parallel to $\hat{\Gamma}_{\mathcal{N}} \cap B_{R_{0}}$.

6) If $\hat{\Gamma}_{\mathcal{N}} \cap B_{R_{0}}=\emptyset$, then $\Lambda_{2}=\{1, \ldots ; n\}$.

7) $\zeta^{i}\left(i \in \Lambda_{2}\right)$ satisfies

i) angle $\left(\zeta^{i}, \hat{\Gamma}_{\mathcal{D}} \cap B_{R_{0}}\right) \geq \alpha^{*}$

ii) $y-s \zeta^{i} \notin(\hat{\Omega} \cup \partial \hat{\Omega})$ for $y \in\left(\hat{\Gamma}_{\mathcal{D}} \cap B_{R_{0}}\right)$, and $0<s<R_{0}$.

8) If angle $\left(\hat{\Gamma}_{i}, \hat{\Gamma}_{j}\right)=\pi\left(i \neq j, \hat{\Gamma}_{i} \cap \hat{\Gamma}_{j} \cap B_{R_{0}} \neq \emptyset\right)$, then $\Lambda_{3}=\{n\}$, otherwise $\Lambda_{3}=\emptyset$.

9) $\zeta^{n}\left(n \in \Lambda_{3}\right)$ satisfies angle $\left(\zeta^{n},\left(\hat{\Gamma}_{i} \cup \hat{\Gamma}_{j}\right) \cap B_{R_{0}}\right) \geq \alpha^{*}$ where $i, j$ are given in Assumption 8). 


\section{Remark.}

i) Let us note that there is such a basis. Some examples how to choose the basis vectors are given in Section 4 .

ii) We can find a constant $\alpha^{*}$ depending only on $n$ and on the geometry of $\partial \Omega$.

In the sequel let $h>0$. We define $E_{i}^{\sigma} y=y+\sigma \zeta^{i}, E_{i}^{\sigma} f(y)=f\left(y+\sigma \zeta^{i}\right)$,

$$
D_{i}^{h} f(y)=\frac{E_{i}^{h} f(y)-f(y)}{h} \quad \text { and } \quad D_{i}^{-h} f(y)=\frac{f(y)-E_{i}^{-h} f(y)}{h}
$$

and we will write $E_{i}^{\sigma} f(y) g(y)$ instead of $\left(E_{i}^{\sigma} f(y)\right) g(y)$.

We set $R=\frac{R_{0}}{8}, B=B_{R} \cap \hat{\Omega}, B^{\prime}=B_{4 R} \cap \hat{\Omega}$, and

$$
\begin{gathered}
\hat{\Omega}_{i}^{h}=\left\{y \in B_{R_{0}}: y \neq x+h \zeta^{i}, x \in B_{R_{0}}\right\} \\
\hat{\Omega}_{i}^{-h}=\left\{y \in B_{R_{0}} \backslash \hat{\Omega}: y=x-h \zeta^{i}, x \in B_{R_{0}} \cap \hat{\Omega}\right\} .
\end{gathered}
$$

Let $\tau_{0}$ be a cut-off function with $\tau_{0} \equiv 1$ in $B, \operatorname{supp} \tau_{0}=B_{4 R}$, and $\left|\nabla \tau_{0}\right| \leq c$, where $c$ depends only on $R_{0}$. By $\tau$ we denote the restriction of $\tau_{0}$ onto $\hat{\Omega} \cup \partial \hat{\Omega}$.

Moreover, we need appropriate extensions of functions into $\hat{\Omega}_{i}^{-h}$ for $i \in \Lambda_{2}$. Let the function $g(y)$ be defined on $\hat{\Omega}$. Let $z_{0} \in \partial \hat{\Omega} \cap B_{R_{0}}$ and $z_{0}-\lambda \zeta^{i} \in \hat{\Omega}_{i}^{-h}$ for $0<\lambda \leq h$. Then we set

$$
g\left(z_{0}-\lambda \zeta^{i}\right)=g\left(z_{0}+\lambda \zeta^{i}\right)
$$

This is an $W^{1,2}$-extension if $g \in W^{1,2}(\hat{\Omega})$. In particular, it holds that $\|g\|_{W^{1,2}\left(\dot{\Omega}_{i}^{-h}\right)} \leq$ $c\|g\|_{W^{1,2}(\dot{\Omega})}$, where the constant $c$ depends only on the data, for $\alpha^{*}$. depends only on $\bar{n}$ and on the geometry of $\partial \Omega$.

Next, we define an appropriate extension of $v=u \circ\left(\phi^{*}\right)^{-1}$ into $\hat{\Omega}_{i}^{-h}$ for $i \in \Lambda_{2}$. Let $y \in \partial \hat{\Omega} \cap \partial \hat{\Omega}_{i}^{-h}, 0<\lambda \leq h$, and $y-\lambda \zeta^{i} \in \hat{\Omega}_{i}^{-h}$. We set

$$
v\left(y-\lambda \zeta^{i}\right)=0
$$

This provides an $W^{1,2}$-extension of $v$, for $i \in \Lambda_{2}$ implies that $\left(\partial \hat{\Omega} \cap \partial \hat{\Omega}_{i}^{-k}\right) \subset \hat{\Gamma}_{\mathcal{D}}$. In particular, it holds for $1 \leq r \leq N$ that

$$
\left\|v^{r}\right\|_{\mathcal{H}^{\frac{3}{2}, 2}\left(\dot{\Omega}_{i}^{-1}\right)} \leq c\left\|v^{r}\right\|_{\mathcal{H}^{\frac{3}{2}, 2}(\dot{\Omega})}
$$

where $c$ and $c^{\prime}$ depend only on the data and $v^{r}$ is the $r$-th component of $v$. Thus, extension (2.2) is an $\mathcal{H}^{\frac{3}{2}, 2}$-extension (cf. [8]).

In what follows we will write $\sum_{i, k, l}$ and $\sum_{r, s}$ instead of $\sum_{i, k, l=1}^{n}$ and $\sum_{r, s=1}^{N}$, respec-

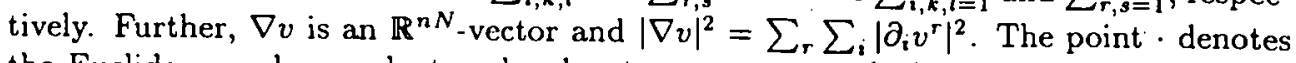
the Euclidean scalar product and $c$ denotes a constant which will be allowed to vary from equation to equation. 


\section{The regularity of the solution}

In this section we prove Theorem 1.1 and Theorem 1.2.

Let $A$ be the matrix whose elements are defined by $a_{i k}=\frac{\partial}{\partial x_{i}}\left(\phi^{* k}\right)$, where $\phi^{* k}$ denotes the $k$-th component of $\phi^{*}(x)$. Let $y=\hat{x}$. In the sequel we only deal with functions defined onto $\hat{\Omega}$. For simplicity we will write $f(y)$ instead of $f\left(\left(\phi^{*}\right)^{-1}(y)\right)$ etc. The function $v=u \circ\left(\phi^{*}\right)^{-1}$ is the weak solution of

$$
-\sum_{i} \widetilde{\partial}_{i} F_{i}(y, \widetilde{\nabla} v)=f(y)+\sum_{i} \widetilde{\partial}_{i} f_{i}(y)
$$

where $\tilde{\partial}_{i} v(y)=\sum_{k} a_{i k}(y) \partial_{k} v(y)$

In detail, $A$ is positive definite, the smallest eigenvalue $\lambda_{0}>0$ depends only on the geometry of $\partial \Omega$, and

$$
a_{i k}(y) \in W^{1, \infty}(\hat{\Omega})
$$

holds. Further, let us note that $v(y) \in\left[W^{1,2}(\hat{\Omega})\right]^{N}$.

We need several propositions.

Proposition 3.1. It holds that

$$
\sup _{0<h<4 R} \int_{B^{\prime}} \tau h\left|D_{i}^{h} \nabla v\right|^{2} d y \leq c \quad \text { for } i \in \Lambda_{1}
$$

where the constant $c$ depends only on $R_{0}$ and the data.

Proof. Let $0<h<4 R$. First, we suppose that $1 \in \Lambda_{1}$ and we prove (3.3) for $i=1$. The Taylor expansion of $F(y, p)\left(p \in \mathbb{R}^{n N}\right)$ entails

$$
\begin{aligned}
& \sum_{r} \sum_{i}\left(\dot{p}^{\prime}-p\right)_{i}^{r} F_{i}^{r}(y, p)=F\left(y, p^{\prime}\right)-F(y, p) \\
& -\sum_{r, s} \sum_{i, k}\left(p^{\prime}-p\right)_{i}^{r}\left(p^{\prime}-p\right)_{k}^{s} \int_{0}^{1}(1-t) F_{i, k}^{r s}\left(y, t p^{\prime}+(1-t) p\right) d t
\end{aligned}
$$

Let

$$
m_{i k}^{r s}(h)=\int_{0}^{1}(1-t) F_{i, k}^{r s}\left(y, t E_{1}^{h} \tilde{\nabla} v+(1-t) \tilde{\nabla} v\right) d t
$$

for $1 \leq i, k \leq n$ and $1 \leq r, s \leq N$. We set $p=\widetilde{\nabla} v$ and $p^{\prime}=E_{1}^{h} \widetilde{\nabla} v$. Thus, $\left(p^{\prime}-p\right)_{i}^{r}=$ $h D_{1}^{h} \tilde{\partial}_{i} v^{r} \equiv \sum_{l} h D_{1}^{h}\left(a_{i l} \partial_{l} v^{r}\right)$ and

$$
\begin{aligned}
& \sum_{r} \sum_{i, l} F_{i}^{r}(y, \tilde{\nabla} v) D_{1}^{h}\left(a_{i l} \partial_{l} v^{r}\right)=\frac{F\left(y, E_{1}^{h} \tilde{\nabla} v\right)-F(y, \tilde{\nabla} v)}{h} \\
& -\sum_{r, s} \sum_{i, k} h\left(\sum_{l} D_{1}^{h}\left(a_{i l} \partial_{l} v^{r}\right)\right)\left(\sum_{l} D_{1}^{h}\left(a_{k l} \partial_{l} v^{s}\right)\right) m_{i k}^{r s}(h) .
\end{aligned}
$$


The function $\varphi=\tau D_{1}^{h} v$ is an admissible test function. Multiplying (3.1) by $\varphi$ yields

$$
\begin{gathered}
\sum_{i, l} \int_{B^{\prime}} F_{i}(y, \tilde{\nabla} v) \cdot \partial_{l}\left(a_{i l} \tau\right) D_{1}^{h} v+\sum_{i, l} \int_{B^{\prime}} F_{i}(y, \tilde{\nabla} v) \cdot\left(a_{i l} \tau\right) \partial_{l} D_{1}^{h} v \\
=\int_{B^{\prime}} \tau f \cdot D_{1}^{h} v-\sum_{i, l} \int_{B^{\prime}} f_{i} \cdot \partial_{l}\left(a_{i l} \tau D_{1}^{h} v\right)
\end{gathered}
$$

where the point - denotes the Euclidean scalar product in $\mathbb{R}^{N}$. Applying (3.5) we obtain

$$
\begin{aligned}
(I)= & \int_{B^{\prime}} \tau \sum_{r_{1} s} \sum_{i, k} h\left(\sum_{l} D_{1}^{h}\left(a_{i l} \partial_{l} v^{r}\right)\right)\left(\sum_{l} D_{1}^{h}\left(a_{k l} \partial_{l} v^{s}\right)\right) m_{i k}^{r s}(h) \\
= & \int_{B^{\prime}} \tau \frac{F\left(y, E_{1}^{h} \tilde{\nabla} v\right)-F(y, \widetilde{\nabla} v)}{h}-\sum_{i, l} \int_{B^{\prime}} \tau F_{i}(y, \tilde{\nabla} v) \cdot D_{1}^{h} a_{i l} \partial_{l} E_{1}^{h} v \\
& +\sum_{i, l} \int_{B^{\prime}} F_{i}(y, \tilde{\nabla} v) \cdot \partial_{l}\left(a_{i l} \tau\right) D_{1}^{h} v-\int_{B^{\prime}} \tau f \cdot D_{1}^{h} v+\sum_{i, l} \int_{B^{\prime}} f_{i} \cdot \partial_{l}\left(a_{i l} \tau D_{1}^{h} v\right) \\
= & (I I)+\ldots+(V I) .
\end{aligned}
$$

The identity $D_{1}^{h}(g \tilde{g})=D_{1}^{h} g E_{1}^{h} \tilde{g}+g D_{1}^{h} \tilde{g}$ yields

$$
\begin{aligned}
(I)= & \int_{B^{\prime}} \tau \sum_{r, s} \sum_{i, k} h\left(\sum_{l}\left(D_{1}^{h} a_{i l} \partial_{l} E_{1}^{h} v^{r}+a_{i l} D_{1}^{h} \partial_{l} v^{r}\right)\right) \\
& \times\left(\sum_{l}\left(D_{1}^{h} a_{k l} \partial_{l} E_{1}^{h} v^{s}+a_{k l} D_{1}^{h} \partial_{l} v^{s}\right)\right) m_{i k}^{r s}(h)
\end{aligned}
$$

By (3.2) and hypothesis (H5) it follows that

$$
\begin{gathered}
\left|\int_{B^{\prime}} \tau \sum_{r, s} \sum_{i, k} h\left(\sum_{l} D_{1}^{h} a_{i l} \partial_{l} E_{1}^{h} v^{r}\right)\left(\sum_{l} D_{1}^{h} a_{k l} \partial_{l} E_{1}^{h} v^{s}\right) m_{i k}^{r s}(h)\right| \\
\leq c h\left\|\nabla E_{1}^{h} v\right\|_{L^{2}\left(B^{\prime}\right)}^{2}
\end{gathered}
$$

and

$$
\begin{gathered}
\left|\int_{B^{\prime}} \tau \sum_{r, s} \sum_{i, k} h\left(\sum_{l} D_{1}^{h} a_{i l} \partial_{l} E_{1}^{h} v^{r}\right)\left(\sum_{l} a_{k l} D_{1}^{h} \partial_{l} v^{s}\right) m_{i k}^{r s}(h)\right| \\
\leq \frac{c h}{\eta}\left\|\nabla E_{1}^{h} v\right\|_{L^{2}\left(B^{\prime}\right)}^{2}+\eta h \int_{B^{\prime}} \tau\left|D_{1}^{h} \nabla v\right|^{2}
\end{gathered}
$$


for $\eta>0$. Hypothesis (H6) entails

$$
\begin{aligned}
\int_{B^{\prime}} \tau & \sum_{r, s} \sum_{i, k} h\left(\sum_{l} a_{i l} D_{1}^{h} \partial_{l} v^{r}\right)\left(\sum_{l} a_{k l} D_{1}^{h} \partial_{l} v^{s}\right) m_{i k}^{r s}(h) \\
& \geq \frac{k_{0}}{2} \int_{B^{\prime}} \tau \sum_{r} \sum_{i} h\left(\sum_{l} a_{i l} D_{1}^{h} \partial_{l} v^{r}\right)^{2} \\
& =\frac{k_{0}}{2} \int_{B^{\prime}} \tau \sum_{r} h D_{1}^{h} \nabla v^{r} \cdot\left(A^{T} A\right) D_{1}^{h} \nabla v^{r} \\
& \geq \frac{k_{0} \lambda_{0}^{2}}{2} \int_{B^{\prime}} \tau h\left|D_{1}^{h} \nabla v\right|^{2} .
\end{aligned}
$$

Altogether we obtain

$$
(I) \geq c \int_{B^{\prime}} \tau h\left|D_{1}^{h} \nabla v\right|^{2}-c h
$$

for a sufficiently small $\eta>0$. Further, using Taylor expansion and summation by parts we get

$$
\begin{aligned}
(I I)= & \int_{B^{\prime}} \tau \frac{F\left(y, E_{1}^{h} \tilde{\nabla} v\right)-F\left(E_{1}^{h} y, E_{1}^{h} \tilde{\nabla} v\right)}{h}+\int_{B^{\prime}} \tau D_{1}^{h} F(y, \tilde{\nabla} v) \\
= & \int_{B^{\prime}} \tau \sum_{k} \zeta^{1 k} \int_{0}^{1} F_{x_{k}}\left(t y+(1-t) E_{1}^{h} y, E_{1}^{h} \tilde{\nabla} v\right) d t d y \\
& +\int_{B^{\prime}} D_{1}^{h}(\tau F(y, \tilde{\nabla} v))-\int_{B^{\prime}} D_{1}^{h} \tau F\left(E_{1}^{h} y, E_{1}^{h} \tilde{\nabla} v\right) \\
= & (I I)_{1}+(I I)_{2}+(I I)_{3}
\end{aligned}
$$

where $\zeta^{1 k}$ denotes the $k$-th component of the basis vector $\zeta^{1}$. Hypotheses (H2) and (H1) entail

$$
\begin{aligned}
\left|(I I)_{1}\right| & \leq c\left(\sum_{k} \sup _{0 \leq t \leq 1}\left\|g_{x_{k}}\left(y+t h \zeta^{1}\right)\right\|_{L^{1}\left(B^{\prime}\right)}+\left\|E_{1}^{h} \tilde{\nabla} v\right\|_{L^{2}\left(B^{\prime}\right)}^{2}\right) \leq c \\
(I I)_{2} & =-h^{-1} \int_{\hat{\boldsymbol{\Omega}}_{1}^{h}} \tau F(y, \tilde{\nabla} v) . \\
\left|(I I)_{3}\right| & \leq c \int_{B^{\prime}}\left(\left|E_{1}^{h} g_{0}\right|+\left|E_{1}^{h} \tilde{\nabla} v\right|^{2}\right) \leq c .
\end{aligned}
$$

By (3.2) and hypotheses (H3) and (H7) we get

$$
\begin{aligned}
|(I I I)| & \leq c\left(\sum_{i}\left\|g_{i}\right\|_{L^{2}\left(B^{\prime}\right)}^{2}+\|\tilde{\nabla} v\|_{L^{2}\left(B^{\prime}\right)}^{2}+\left\|\nabla E_{1}^{h} v\right\|_{L^{2}\left(B^{\prime}\right)}^{2}\right) \leq c \\
|(I V)| & \leq c\left(\sum_{i}\left\|g_{i}\right\|_{L^{2}\left(B^{\prime}\right)}^{2}+\|\tilde{\nabla} v\|_{L^{2}\left(B^{\prime}\right)}^{2}+\left\|D_{1}^{h} v\right\|_{L^{2}\left(B^{\prime}\right)}^{2}\right) \leq c \\
|(V)| & \leq c\left(\|f\|_{L^{2}\left(B^{\prime}\right)}^{2}+\left\|D_{1}^{h} v\right\|_{L^{2}\left(B^{\prime}\right)}^{2}\right) \leq c .
\end{aligned}
$$


Next, summation by parts yields

$$
\begin{aligned}
(V I) & =\sum_{i, l} \int_{B^{\prime}} f_{i} \cdot \partial_{l}\left(\tau a_{i l}\right) D_{1}^{h} v-\sum_{i, l} \int_{B^{\prime}} D_{1}^{h}\left(\tau a_{i l} f_{i}\right) \cdot \partial_{l} E_{1}^{h} v+\sum_{i, l} \int_{B^{\prime}} D_{1}^{h}\left(\tau a_{i l} f_{i} \cdot \partial_{l} v\right) \\
& =(V I)_{1}+(V I)_{2}+(V I)_{3} .
\end{aligned}
$$

Due to (3.2) and hypothesis (H7) we obtain

$$
\begin{aligned}
& \left|(V I)_{1}\right| \leq c\left(\sum_{i}\left\|f_{i}\right\|_{L^{2}\left(B^{\prime}\right)}^{2}+\left\|D_{1}^{h} v\right\|_{L^{2}\left(B^{\prime}\right)}^{2}\right) \leq c \\
& \left|(V I)_{2}\right| \leq c\left(\sum_{i}\left\|f_{i}\right\|_{L^{2}\left(B^{\prime}\right)}^{2}+\sum_{i}\left\|D_{1}^{h} f_{i}\right\|_{L^{2}\left(B^{\prime}\right)}^{2}+\left\|\nabla E_{1}^{h} v\right\|_{L^{2}\left(B^{\prime}\right)}^{2}\right) \leq c .
\end{aligned}
$$

Applying hypothesis (H1) we get for $\eta>0$

$$
\begin{aligned}
\left|(V I)_{3}\right| & =\left|\frac{1}{h} \sum_{i, l} \int_{\hat{\Omega}_{1}^{h}} \tau a_{i l} f_{i} \cdot \partial_{l} v\right| \\
& \leq \frac{c}{\eta h}\left|\hat{\Omega}_{1}^{h}\right| \sum_{i}\left\|f_{i}\right\|_{L^{\infty}\left(\hat{\Omega}_{1}^{h}\right)}^{2}+\frac{\eta}{h} \int_{\hat{\Omega}_{1}^{h}} \tau|\tilde{\nabla} v|^{2} \\
& \leq c+\frac{\eta}{c_{0}^{\prime} h} \int_{\hat{\Omega}_{1}^{h}} \tau F(y, \tilde{\nabla} v) .
\end{aligned}
$$

Let $\eta=\frac{c_{0}^{\prime}}{2}$. Then $(3.6),(3.7)$, and hypothesis $(\mathrm{H} 1)$ yield

$$
(I I)_{2}+\left|(V I)_{3}\right| \leq c-\frac{1}{2 h} \int_{\hat{\Omega}_{1}^{h}} \tau F(y, \widetilde{\nabla} v) \leq c-\frac{c_{0}}{2 h}\left|\hat{\Omega}_{1}^{h}\right| \leq c .
$$

Altogether we obtain assertion (3.3) for $i=1$. Finally, let us note that the proof of (3.3) for arbitrary $i \in \Lambda_{1}$ follows in the same way

Proposition 3.2. There exists a constant $c$ depending only on $R_{0}$ and the data such that

$$
\sup _{0<h<4 R} \int_{B^{\prime}} \tau h\left|D_{i}^{-h} \nabla v\right|^{2} d y \leq c \quad \text { for } i \in \Lambda_{2} .
$$

Proof. Let $0<h<4 R$. We give the proof of (3.8) for some fixed number $i \in \Lambda_{2}$, say $i=1$.

First, we extend $v$ into $\hat{\Omega}_{1}^{-h}$ by using (2.2), and the functions $F(\cdot, p), g_{0}, \tau, a_{i k}(1 \leq$ $i, k \leq n)$ by using (2.1). Now, let us verify that $\varphi=-\tau D_{1}^{-h} v$ is an admissible test function. The conditions on $\zeta^{i}\left(i \in \Lambda_{2}\right)$ imply that $y-h \zeta^{1} \notin \hat{\Omega} \cup \partial \hat{\Omega}$ for $y \in \hat{\Gamma}_{\mathcal{D}} \cap B^{\prime}$. Hence, the extension (2.2) yield

$$
v\left(y-h \zeta^{1}\right)=0 \quad \text { for } y \in \hat{\Gamma}_{\mathcal{D}} \cap B^{\prime}
$$


thus

$$
\varphi(y)=\tau h^{-1}\left(v\left(y-h \zeta^{1}\right)-v(y)\right)=0 \quad \text { for } y \in \hat{\Gamma}_{\mathcal{D}} \cap B^{\prime}
$$

Multiplying (3.1) by $\varphi$ and integrating over $\hat{\Omega}$ we get

$$
\begin{aligned}
& -\int_{B^{\prime}} \tau f \cdot D_{1}^{-h} v+\sum_{i, l} \int_{B^{\prime}} f_{i} \cdot \partial_{l}\left(a_{i l} \tau D_{1}^{-h} v\right)+\sum_{i, l} \int_{B^{\prime}} F_{i}(y, \tilde{\nabla} v) \cdot \partial_{l}\left(a_{i l} \tau\right) D_{1}^{-h} v \\
& =-\sum_{i, l} \int_{B^{\prime}} F_{i}(y, \tilde{\nabla} v) \cdot\left(\tau a_{i l}\right) \partial_{l} D_{1}^{-h} v \\
& =\sum_{i, l} \int_{B^{\prime}} \tau F_{i}(y, \tilde{\nabla} v) \cdot\left[-D_{1}^{-h}\left(a_{i l} \partial_{l} v\right)+D_{1}^{-h} a_{i l} E_{1}^{-h} \partial_{l} v\right]
\end{aligned}
$$

where we have used the identity $D_{1}^{-h}(g \tilde{g})=D_{1}^{-h} g E_{1}^{-h} \tilde{g}+g D_{1}^{-h} \tilde{g}$. The Taylor expansion of $F(y, \cdot)$ yields

$$
\begin{aligned}
\sum_{r} \sum_{i} & \left(p^{\prime}-p\right)_{i}^{r} F_{i}^{r}(y, p) \\
= & F\left(y, p^{\prime}\right)-F(y, p) \\
& -\sum_{r, s} \sum_{i, k}\left(p^{\prime}-p\right)_{i}^{r}\left(p^{\prime}-p\right)_{k}^{s} \int_{0}^{1}(1-t) F_{i, k}^{r s}\left(y, t p^{\prime}+(1-t) p\right) d t
\end{aligned}
$$

We set

$$
m_{i k}^{r s}(-h)=\int_{0}^{1}(1-t) F_{i, k}^{r s}\left(y, t E_{1}^{-h} \tilde{\nabla} v+(1-t) \widetilde{\nabla} v\right) d t
$$

for $1 \leq i, k \leq n$ and $1 \leq r, s \leq N$. Let us put $p=\widetilde{\nabla} v$ and $p^{\prime}=E_{1}^{-\imath} \widetilde{\nabla} v$. Then we obtain

$$
\begin{aligned}
-\sum_{r} & \sum_{i, l} F_{i}^{r}(y, \tilde{\nabla} v) D_{1}^{-h}\left(a_{i l} \partial_{l} v^{r}\right) \\
= & \frac{1}{h}\left(F\left(y, E_{1}^{-h} \tilde{\nabla} v\right)-F(y, \tilde{\nabla} v)\right) \\
& -\sum_{r, s} \sum_{i, k} h\left(\sum_{l} D_{1}^{-h}\left(a_{i l} \partial_{l} v^{r}\right)\right)\left(\sum_{l} D_{1}^{-h}\left(a_{k l} \partial_{l} v^{s}\right)\right) m_{i k}^{r s}(-h)
\end{aligned}
$$

Thus, (3.9) yields

$$
(I)=\int_{B^{\prime}} \tau h \sum_{r, s} \sum_{i, k}\left(\sum_{l} D_{1}^{-h}\left(a_{i l} \partial_{l} v^{r}\right)\right)\left(\sum_{l} D_{1}^{-h}\left(a_{k l} \partial_{l} v^{s}\right)\right) m_{i k}^{r s}(-h)
$$




$$
\begin{aligned}
= & \int_{B^{\prime}} \tau h^{-1}\left(F\left(y, E_{1}^{-h} \tilde{\nabla} v\right)-F(y, \tilde{\nabla} v)\right) \\
& +\sum_{i, l} \int_{B^{\prime}} \tau F_{i}(y, \tilde{\nabla} v) \cdot D_{1}^{-h} a_{i l} \partial_{l} E_{1}^{-h} v \\
& -\sum_{i, l} \int_{B^{\prime}} F_{i}(y, \tilde{\nabla} v) \cdot \partial_{l}\left(a_{i l} \tau\right) D_{1}^{-h} v \\
& +\int_{B^{\prime}} \tau f \cdot D_{1}^{-h} v \\
& -\sum_{i, l} \int_{B^{\prime}} f_{i} \cdot \partial_{l}\left(a_{i l} \tau D_{1}^{-h} v\right) \\
= & (I I I)+\ldots+(V I) .
\end{aligned}
$$

Hypothesis (H6) entails

$$
(I) \geq \frac{k_{0}}{2} \int_{B^{\prime}} \tau h D_{1}^{-h} \tilde{\nabla} v \cdot D_{1}^{-h} \tilde{\nabla} v=\frac{k_{0}}{2} \int_{B^{\prime}} \tau h \sum_{r}\left|D_{1}^{-h}\left(A \nabla v^{r}\right)\right|^{2} .
$$

We use

$$
\begin{gathered}
\int_{B^{\prime}} \tau h A D_{1}^{-h} \nabla v^{r} \cdot A D_{1}^{-h} \nabla v^{r} \geq \lambda_{0}^{2} \int_{B^{\prime}} \tau h\left|D_{1}^{-h} \nabla v^{r}\right|^{2} \\
\int_{B^{\prime}} \tau h\left(D_{1}^{-h} A\right) \nabla E_{1}^{-h} v^{r} \cdot\left(D_{1}^{-h} A\right) \nabla E_{1}^{-h} v^{r} \leq c \int_{B^{\prime}} \tau h\left|\nabla E_{1}^{-h} v^{r}\right|^{2} \leq c \\
2 \int_{B^{\prime}} \tau h\left(D_{1}^{-h} A\right) \nabla E_{1}^{-h} v^{r} \cdot A D_{1}^{-h} \nabla v^{r} \leq \frac{c}{\eta} \int_{B^{\prime}} \tau h\left|\nabla E_{1}^{-h} v^{r}\right|^{2}+\eta \int_{B^{\prime}} \tau h\left|D_{1}^{-h} \nabla v^{r}\right|^{2}
\end{gathered}
$$

for $\eta>0$. Putting $\eta=\frac{k_{0} \lambda_{0}^{2}}{4}$ it follows that

$$
(I) \geq \frac{k_{0} \lambda_{0}^{2}}{4} \int_{B^{\prime}} \tau h\left|D_{1}^{-h} \nabla v\right|^{2}-c .
$$

Next,

$$
\begin{aligned}
(I I) & =-\int_{B^{\prime}} \tau D_{1}^{-h} F(y, \tilde{\nabla} v)+\int_{B^{\prime}} \tau h^{-1}\left(F\left(y, E_{1}^{-h} \tilde{\nabla} v\right)-F\left(E_{1}^{-h} y, E_{1}^{-h} \tilde{\nabla} v\right)\right) \\
& =(I I)_{1}+(I I)_{2} .
\end{aligned}
$$

Summation by parts entails

$$
\begin{aligned}
(I I)_{1} & =-\int_{B^{\prime} \cup B^{\prime \prime}} \tau D_{1}^{-h} F(y, \widetilde{\nabla} v) \\
& =-\int_{B^{\prime} \cup B^{\prime \prime}} D_{1}^{-h}(\tau F(y, \widetilde{\nabla} v))+\int_{B^{\prime} \cup B^{\prime \prime}} D_{1}^{-h} \tau F\left(E_{1}^{-h} y, E_{1}^{-h} \tilde{\nabla} v\right) \\
& =(I I)_{11}+(I I)_{12}
\end{aligned}
$$


where

$$
B^{\prime \prime}=\left\{y \in B_{R_{0}} \backslash B^{\prime}: y=x+h \zeta^{1}, x \in B^{\prime}\right\} \text {. }
$$

The extensions (2.1) and (2.2) entail

$$
\left|(I I)_{11}\right|=\frac{1}{h}\left|\int_{\dot{\Omega}_{1}^{-h}} \tau F(y, \widetilde{\nabla} v)\right| \leq \frac{1}{h} \int_{\dot{\Omega}_{1}^{-h}}\left|g_{0}\right| \leq\left\|g_{0}\right\|_{L^{\infty}\left(\dot{\Omega}_{1}^{h}\right)} \frac{1}{h}\left|\hat{\Omega}_{1}^{-h}\right| \leq c .
$$

Further, using hypothesis (H1) we obtain

$$
\left|(I I)_{12}\right| \leq c \int_{B^{\prime}}\left|F\left(E_{1}^{-h} y, E_{1}^{-h} \tilde{\nabla} v\right)\right| \leq c \int_{B^{\prime}}\left(\left|E_{1}^{-h} g_{0}\right|+\left|E_{1}^{-h} \tilde{\nabla} v\right|^{2}\right) \leq c .
$$

Let $\zeta^{1 k}$ be the $k$-th component of the basis vector $\zeta^{1}$. Hypothesis (H2) and the Taylor expansion entail

$$
\begin{aligned}
\left|(I I)_{2}\right| & \leq \int_{B^{\prime}} \tau \sum_{k}\left|\zeta^{1 k}\right| \int_{0}^{1}\left|F_{x_{k}}\left(t y+(1-t) E_{1}^{-h} y, E_{1}^{-h} \tilde{\nabla} v\right)\right| d t d y \\
& \leq c\left(\sum_{k} \sup _{0 \leq t \leq 1}\left\|g_{x_{k}}\left(y-t h \zeta^{1}\right)\right\|_{L^{1}\left(B^{\prime}\right)}+\left\|E_{1}^{-h} \tilde{\nabla} v\right\|_{L^{2}\left(B^{\prime}\right)}^{2}\right) \\
& \leq c .
\end{aligned}
$$

By (3.2) and Hypotheses (H3) and (H7) we get

$$
\begin{gathered}
|(I I I)| \leq c\left(\sum_{i}\left\|g_{i}\right\|_{L^{2}\left(B^{\prime}\right)}^{2}+\|\tilde{\nabla} v\|_{L^{2}\left(B^{\prime}\right)}^{2}+\left\|\nabla E_{1}^{-h} v\right\|_{L^{2}\left(B^{\prime}\right)}^{2}\right) \leq c \\
|(I V)| \leq c\left(\sum_{i}\left\|g_{i}\right\|_{L^{2}\left(B^{\prime}\right)}^{2}+\|\tilde{\nabla} v\|_{L^{2}\left(B^{\prime}\right)}^{2}+\left\|D_{1}^{-h} v\right\|_{L^{2}\left(B^{\prime}\right)}^{2}\right) \leq c \\
|(V)| \leq c\left(\|f\|_{L^{2}\left(B^{\prime}\right)}^{2}+\left\|D_{1}^{-h} v\right\|_{L^{2}\left(B^{\prime}\right)}^{2}\right) \leq c .
\end{gathered}
$$

Next,

$$
\begin{aligned}
(V I) & =-\sum_{i, l} \int_{B^{\prime}} f_{i} \cdot \partial_{l}\left(a_{i l} \tau\right) D_{1}^{-h} v-\sum_{i, l} \int_{B^{\prime}} \tau a_{i l} f_{i} \cdot D_{1}^{-h} \partial_{l} v \\
& =(V I)_{1}+(V I)_{2} .
\end{aligned}
$$

Due to (3.2) and Hypothesis (H1)

$$
\left|(V I)_{1}\right| \leq c\left(\sum_{i}\left\|f_{i}\right\|_{L^{2}\left(B^{\prime}\right)}^{2}+\left\|D_{1}^{-h} v\right\|_{L^{2}\left(B^{\prime}\right)}^{2}\right) \leq c
$$

follows. Using summation by parts we obtain

$$
\begin{aligned}
(V I)_{2} & =-\sum_{i, l} \int_{B^{\prime} \cup B^{\prime \prime}} \tau a_{i l} f_{i} \cdot D_{1}^{-h} \partial_{l} v \\
& =\sum_{i, l} \int_{B^{\prime} \cup B^{\prime \prime}} D_{1}^{-h}\left(\tau a_{i l} f_{i}\right) \partial_{l} E_{1}^{-h} v-\sum_{i, l} \int_{B^{\prime} \cup B^{\prime \prime}} D_{1}^{-h}\left(\tau a_{i l} f_{i} \partial_{l} v\right) \\
& =(V I)_{3}+(V I)_{4} .
\end{aligned}
$$


In view of hypothesis (H7) we get

$$
\begin{aligned}
\left|(V I)_{3}\right| & =\sum_{i, l} \int_{B^{\prime}}\left(D_{1}^{-h}\left(\tau a_{i l}\right) f_{i}+E_{1}^{-h}\left(\tau a_{i l}\right) D_{1}^{-h} f_{i}\right) \partial_{l} E_{1}^{-h} v \\
& \leq c\left(\sum_{i}\left\|f_{i}\right\|_{L^{2}\left(B^{\prime}\right)}^{2}+\sum_{i}\left\|D_{1}^{-h} f_{i}\right\|_{L^{2}\left(B^{\prime}\right)}^{2}+\left\|\nabla E_{1}^{-h} v\right\|_{L^{2}\left(B^{\prime}\right)}^{2}\right) \\
& \leq c .
\end{aligned}
$$

The extension (2.2) yields $\partial_{l} v=0$ in $\hat{\Omega}_{1}^{-h}$. This implies that

$$
(V I)_{4}=\frac{1}{h} \sum_{i, l} \int_{\hat{\Omega}_{1}^{-h}} \tau a_{i l} f_{i} \partial_{l} v=0 .
$$

Thus, the assertion follows

Proposition 3.3. Let $\Lambda_{3}=\{n\}$ and $0<\delta<\frac{1}{2}$. Then there exists a constant $c$ depending only on $R_{0}, \delta$, and the data such that

$$
\sup _{0<h<4 R} \int_{B} h^{1+\delta}\left|D_{n}^{h} \nabla v\right|^{2} d y \leq c .
$$
series.

The proof of this proposition follows as in [8] using (3.1), (3.3), (3.8), and Fourier

Now, we are able to prove the main results.

Proof of Theorem 1.1. a) Recall that $\Omega_{\eta}=\{x \in \Omega: \operatorname{dist}(x, \partial \Omega) \geq \eta\}$ and note that the basis vectors $\zeta^{i}$ fulfil angle $\left(\zeta^{i}, \zeta^{j}\right) \geq \alpha^{*}$ for $1 \leq i<j \leq n$, where the constant $\alpha^{*}$ depends only on the geometry of $\partial \Omega$. It holds that $\tau \equiv 1$ in $B$. Thus, (3.3), (3.8), and (3.10) yield for all $\delta \in\left(0, \frac{1}{2}\right)$

$$
\sup _{\substack{n>0 \\ 0<|z|<\eta}} \int_{\left(\left(\phi^{\bullet}\right)^{-1}(B)\right)_{\eta}} \frac{|\nabla u(x+z)-\nabla u(x)|^{2}}{|z|^{1-\delta}} d x \leq c
$$

where the constant $c$ depends only on the data, $\delta$, and on $R_{0}$. Further, let us note that $R_{0}$ depends only on the shape of $\partial \Omega$. that

Next, there are a finite set of points $\left\{\hat{P}_{1}, \ldots, \hat{P}_{k}\right\}$ and a set of balls $B_{R_{i}}\left(\hat{P}_{i}\right)$ such

$$
\partial \Omega \subset \bigcup_{i=1}^{k}\left(B^{i} \cap \partial \Omega\right), \quad \text { where } B^{i}=\left(\phi^{*}\right)^{-1}\left(B_{R_{i}}\left(\hat{P}_{i}\right)\right)
$$

and $\hat{P}_{i}$ is the only vertex of $\partial \hat{\Omega}$ in $B_{R_{i}}\left(\hat{P}_{i}\right)$ or $B_{R_{i}}\left(\hat{P}_{i}\right) \cap \partial \hat{\Omega}$ contains no vertex of $\partial \hat{\Omega}$. Further, the radii $R_{i} \quad(1 \leq i \leq k)$ depend only on the data, for they are determined by the geometry of $\Omega$. Thus,

$$
u \in\left[\mathcal{H}^{\frac{3}{2}-\frac{\delta}{2}, 2}(\Omega)\right]^{N} \quad \text { for } \delta \in\left(0, \frac{1}{2}\right)
$$

follows. The imbedding theorem of Nikolskii spaces into Sobolev spaces (cf. [1])

$$
\mathcal{H}^{s, p}(\Omega) \rightarrow W^{s-\varepsilon, p}(\Omega) \quad \text { for } \varepsilon>0
$$

entails $u \in\left[W^{s, 2}(\Omega)\right]^{N}$ for all $s<\frac{3}{2}$. This yields assertion (1.1).

b) Using (3.3) and (3.8) we get (3.11) for $\delta=0$. Proceeding as above we obtain $u \in\left[\mathcal{H}^{\frac{3}{2}, 2}(\Omega)\right]^{N}$ 
Proof of Theorem 1.2. We only sketch the proof. Assumption (1.3) yields $f \in$ $L^{q}(\Omega)$ and $f_{i} \in L^{2 q}(\Omega)$ for some $q>\frac{n}{2}$. Now, $N=1$ holds. Following [13] we see that $u \in C^{0, \alpha}(\bar{\Omega})$ for some $\alpha>0$. Thus, we can proceed as in [8]. The Hölder continuity and the equation yield

$$
\int_{B_{r}\left(y_{0}\right) \cap \tilde{\Omega}} \frac{|\nabla v(y)|^{2}}{\left|y-y_{0}\right|^{n-2+2 \varepsilon}} d y \leq c
$$

for some $\varepsilon>0$. Replacing the test functions $\varphi$ by $r^{-\varepsilon} \varphi$ in Propositions 3.1 and 3.2 and recalling the proof of Proposition 3.3 we get

$$
\int_{B_{r}(\dot{P}) \cap \dot{\Omega}} r^{3-\varepsilon-n}\left|h^{\frac{1+6}{2}} D_{i}^{h} \nabla v\right|^{2} \leq c
$$

for $1 \leq i \leq n, 0<r \leq \frac{R_{0}}{8}$ and $0<\delta<\frac{1}{2}$. Applying an imbedding theorem of Morrey-Nikolskii type we obtain the assertion

\section{Examples}

In this section we give some explicit examples of the index sets $\Lambda_{1}, \Lambda_{2}, \Lambda_{3}$, and the basis vectors $\zeta^{1}, \ldots, \zeta^{n}$.

Let $\Omega \subset \mathbb{R}^{3}$ be a polyhedron. We consider three typical situations: an edge of $\partial \Omega$ (Example 1), the case when angle $\left(\Gamma_{\mathcal{D}}, \Gamma_{\mathcal{N}}\right)=\pi$ (Example 2), and a corner point (Example 3).

Let $P=(0,0,0)^{T}, B_{R_{0}}=\left\{y:|y|<\frac{1}{2}\right\}$, and let $e_{k} \quad(1 \leq k \leq 3)$ be the $k$-th unit vector in $\mathbb{R}^{3}$.

Example 1: Let

$$
\begin{aligned}
& \Gamma_{*}^{1}=\left\{y \in B_{R_{0}}: y_{1}=0, y_{3}>0\right\} \\
& \Gamma_{*}^{2}=\left\{y \in B_{R_{0}}: y_{3}=0, y_{1}>0\right\}
\end{aligned}
$$

and

$$
\Omega \cap B_{1}=\left\{y \in B_{1}: y_{1}>0, y_{3}>0\right\} .
$$

Case 1: $\Gamma_{\mathcal{D}} \cap B_{R_{0}}=\overline{\Gamma^{1}}$ and $\Gamma_{\mathcal{N}} \cap B_{R_{0}}=\Gamma_{*}^{2}$. Let us put $\zeta^{1}=e_{2}$ and $\zeta^{2}=e_{3}$. Then $\zeta^{1}$ and $\zeta^{2}$ are parallel to $\Gamma_{\mathcal{D}} \cap B_{R_{0}}$, thus, $\Lambda_{1}=\{1,2\}$. Next, we put $\Lambda_{2}=\{3\}$. We must choose $\zeta^{3}$ such that $\zeta^{3}$ is parallel to $\Gamma_{\mathcal{N}} \cap B_{R_{0}}$ and angle $\left(\zeta^{3}, \Gamma_{\mathcal{D}} \cap B_{R_{0}}\right) \geq \alpha^{*}$ for some suitable large constant $\alpha^{*}>0$ (i.e., $\alpha^{*} \sim \operatorname{angle}\left(\Gamma_{*}^{1}, \Gamma_{*}^{2}\right)$ ). Thus, let $\zeta^{3}=e_{3}$.

Case 2: $\Gamma_{\mathcal{D}} \cap B_{R_{0}}=\emptyset$ and $\Gamma_{\mathcal{N}} \cap B_{R_{0}}=\overline{\Gamma_{*}^{1}} \cup \overline{\Gamma_{*}^{2}}$. It holds that $\Lambda_{1}=\{1,2,3\}$. We must choose $\zeta^{i}(1 \leq i \leq 3)$ such that

i) $y+s \zeta^{i} \in \bar{\Omega}$ for $y \in \partial \Omega \cap B_{R_{0}}$ and $0<s<R_{0}$

ii) angle $\left(\zeta^{i}, \zeta^{j}\right) \geq \alpha^{*}$ for $1 \leq i<j \leq 3$ and some suitable constant $\alpha^{*}>0$.

Thus, let $\zeta^{i}=e_{i}$ for $1 \leq i \leq 3$.

Case 3: $\Gamma_{\mathcal{D}} \cap B_{R_{0}}=\overline{\Gamma_{0}^{!}} \cup \overline{\Gamma^{2}}$ and $\Gamma_{\mathcal{N}} \cap B_{R_{0}}=\emptyset$. Now, it holds that $\Lambda_{2}=\{1,2,3\}$. The basis vectors $\zeta^{i}(1 \leq i \leq 3)$ must fulfil 
i) $y+s \zeta^{i} \in \bar{\Omega}$ for $y \in \partial \Omega \cap B_{R_{0}}$ and $0<s<R_{0}$

ii) angle $\left(\zeta^{i}, \zeta^{j}\right) \geq \alpha^{*}$ for $1 \leq i<j \leq 3$ and $\alpha^{*}>0$

iii) angle $\left(\zeta^{i}, \Gamma_{\mathcal{D}} \cap B_{R_{0}}\right) \geq \alpha^{*}$

where $\alpha^{*}>0$ is suitable. Thus, let $\zeta^{1}=\frac{\sqrt{3}}{2} e_{1}+\frac{1}{2} e_{3}, \zeta^{2}=\frac{1}{2} e_{1}+\frac{\sqrt{3}}{2} e_{3}$, and $\zeta^{3}=$ $\frac{1}{3} e_{2}+\frac{2}{3}\left(e_{1}+e_{3}\right)$.

Example 2. Let

$$
\Omega \cap B_{R_{0}}=\left\{y \in B_{R_{0}}: y_{3}>0\right\}
$$

and

$$
\begin{aligned}
& \Gamma_{\mathcal{D}} \cap B_{R_{0}}=\left\{y \in B_{R_{0}}: y_{3}=0, y_{1} \geq 0\right\} \\
& \Gamma_{\mathcal{N}} \cap B_{R_{0}}=\left\{y \in B_{R_{0}}: y_{3}=0, y_{1}<0\right\} .
\end{aligned}
$$

We choose $\zeta^{1}=e_{1}$ and $\zeta^{2}=e_{2}$. Then $y+s \zeta^{i} \in \Gamma_{\mathcal{D}} \cap B_{R_{0}}$ holds for $y \in \Gamma_{\mathcal{D}} \cap B_{R_{0}}$, $s>0$, and $y+s \zeta^{i} \in B_{R_{0}}$. Thus, $\Lambda_{1}=\{1,2\}$. Further, $\Lambda_{2}=\emptyset$ and $\Lambda_{3}=\{3\}$. Let us put $\zeta^{3}=e_{3}$.

Example 3. Let $\Omega=[0,1]^{3}$.

Case 1: $\Gamma_{\mathcal{D}}=\left\{y \in \partial \Omega: y_{3}=0\right\}$ and $\Gamma_{\mathcal{N}}=\partial \Omega \backslash \Gamma_{\mathcal{D}}$. The two vectors $e_{1}$ and $e_{2}$ are parallel to $\Gamma_{\mathcal{D}} \cap B_{R_{0}}$ and $e_{3}$ is parallel to $\Gamma_{\mathcal{N}} \cap B_{R_{0}}$. Thus, let $\Lambda_{1}=\{1,2\}, \zeta^{1}=e_{1}$, $\zeta^{2}=e_{2}, \Lambda_{2}=\{3\}$, and $\zeta^{3}=e_{3}$.

Case 2: $\Gamma_{\mathcal{D}}=\left\{y \in \partial \Omega: y_{2}=0 \vee y_{3}=0\right\}$ and $\Gamma_{\mathcal{N}}=\partial \Omega \backslash \Gamma_{\mathcal{D}}$. Now, $e_{1}$ is parallel to $\Gamma_{\mathcal{D}} \cap B_{R_{0}}$, thus, $\Lambda_{1}=\{1\}$ and $\zeta^{1}=e_{1}$. Further, the two vectors $e_{2}$ and $e_{3}$ are parallel to $\Gamma_{\mathcal{N}} \cap B_{R_{0}}$, thus, $\Lambda_{2}=\{2,3\}$. We must choose $\zeta^{i}(i=2,3)$ such that

i) angle $\left(\zeta^{i}, \Gamma_{\mathcal{D}} \cap B_{R_{0}}\right) \geq \alpha^{*}$

ii) angle $\left(\zeta^{2}, \zeta^{3}\right) \geq \alpha^{*}$

for some suitable constant $\alpha^{*}>0$. Thus, let $\zeta^{2}=\frac{\sqrt{3}}{2} e_{2}+\frac{1}{2} e_{3}$ and $\zeta^{3}=\frac{1}{2} e_{2}+\frac{\sqrt{3}}{2} e_{3}$.

Case S: $\Gamma_{\mathcal{D}}=\emptyset$ and $\Gamma_{\mathcal{N}}=\partial \Omega$. It holds that $\Lambda_{1}=\{1,2,3\}$. Let $\zeta^{i}=e_{i}$ for $1 \leq i \leq 3$.

Case 4: $\Gamma_{\mathcal{D}}=\partial \Omega$ and $\Gamma_{\mathcal{N}}=\emptyset$. Now, it holds that $\Lambda_{2}=\{1,2,3\}$. We choose $\zeta^{i}(1 \leq i \leq 3)$ such that

i) angle $\left(\zeta^{i}, \Gamma_{\mathcal{D}} \cap B_{R_{0}}\right) \geq \alpha^{*}$

ii) angle $\left(\zeta^{i}, \zeta^{j}\right) \geq \alpha^{*}$ for $1 \leq i<j \leq 3$ and $\alpha^{*}>0$

iii) $y+s \zeta^{i} \in \Omega$ for $y \in \partial \Omega \cap B_{R_{0}}$ and $0<s<R_{0}$

where $\alpha^{*}>0$ is suitable. 


\section{References}

[1] Adams, R. A.: Sobolev Spaces. New York et al.: Academic Press 1975.

[2] Banasiak, J.: On asymptotics of solutions of elliptic mixed boundary value problems of second-order in domains with vanishing edges. Siam J. Math. Anal. 23 (1992), 1117 1124.

[3] Banasiak, J.: A counterexample in the theory of mixed boundary value problems for elliptic equations in non-smooth domains. Demonstr. Math. 26 (1993), $327-335$.

[4] Banasiak, J. and G. F. Roach: On mixed boundary value problems of Dirichlet obliquederivative type in plane domains with piecewise differentiable boundary. J. Diff. Equ. 79 (1989), $111-131$.

[5] Borsuk, M. V.: Estimates of solutions of the Dirichlet problem for a quasilinear nondivergence elliptic equation of second order near a corner boundary point. St. Petersbg. Math. J. 3 (1992), $1281-1302$.

[6] Borsuk, M. V.: Behaviour of solutions of the Dirichlet problem for a second-order quasilinear elliptic equation of general form close to a corner point. Ukr. Math. J. 44 (1992), $149-155$.

[7] Dauge, M.: Elliptic Boundary Value Problems on Corner Domains. Lect. Notes Math. 1341 (1988), 1 - 257.

[8] Ebmeyer, C. and J. Frehse: Mixed boundary value problems for nonlinear elliptic equations in multidimensional non-smooth domains. Math. Nachr. 203 (1999) (to appear).

[9] Grisvard, P.: Elliptic Problems in Nonsmooth Domains (Pitman Advanced Publishing Program). Boston - London - Melbourne: Pitman 1985.

[10] Grisvard, P.: Edge behavior of the solution of an elliptic problem. Math. Nachr. 132 (1987), 281 - 299.

[11] Kondrat'ev, V. A.: Boundary value problems for elliptic equations in domains with conical and angular points. Trans. Moscow Math. Soc. 16 (1967), $227-313$.

[12] Koslov, V. and V. Maz'ya: Angle singularities of solutions to the Neumann problem for the two-dimensional Riccati's equation. Asymptotic Anal. 19 (1999), 57 - 79.

[13] Ladyzhenskaya, O. A. and N. N.Uraltseva: Linear and Quasilinear Elliptic Equations. New York - London: Acad. Press 1968.

[14] Maz'ja, V. and J. Rossmann: On the behaviour of solutions to the Dirichlet problem for second order elliptic equations near edges and polyhedral vertices with critical angles. Z. Anal. Anw. 13 (1994), $19-47$.

[15] Miersemann, E.: Asymptotic expansions of solutions of the Dirichlet problem for quasilinear elliptic equations of second order near a conical point. Math. Nachr. 135 (1988), $239-274$.

[16] Morrey, C. B.: Multiple Integrals in the Calculus of Variations. Berlin - Heidelberg - New York: Springer-Verlag 1966.

[17] Petersdorff, T. v. and E. P. Stephan: Decompositions in edge and corner singularities for the solution of the Dirichlet problem of the Laplacion in a polyhedron. Math. Nachr. 149 (1990), $71-104$.

Received 08.07.1998; in revised form 22.03.1999 